\title{
EFEITO DE BIOPROTETORES EM PATÓGENOS DE SEMENTES E NA EMERGÊNCIA E RENDIMENTO DE GRÃOS DE MILHO
}

\author{
WILMAR C. LUZ* \\ Embrapa Trigo, Cx. Postal 451, CEP 99001-970 Passo Fundo, RS, Brasil, e.mail:wilmar@cnpt.embrapa.br
}

(Aceito para publicação em 05/12/2000)

LUZ, W.C. da. Efeito de bioprotetores em patógenos de sementes e na emergência e rendimento de grãos de milho. Fitopatologia Brasileira 26:16-20. 2001.

\section{RESUMO}

Um experimento de laboratório e dois de campo, conduzidos nas localidades de Passo Fundo, RS, e Pato Branco, PR, foram realizados com o objetivo de avaliar os efeitos da microbiolização de sementes sobre os patógenos de sementes e sobre a germinação e rendimento de grãos de milho (Zea mays). Em laboratório, a maioria dos bioprotetores reduziu significativamente o nível de patógenos de sementes. Nos experimentos de campo, no ano de 1997, em Passo Fundo, RS, somente Trichoderma harzianum (T-22) melhorou significativamente a emergência e o rendimento de grãos do milho. Todos os bioprotetores melhoraram significativamente a emergência em Pato Branco (PR). Paenibacillus macerans (144), T. harzianum (T-22), Flavimonas oryzihabitans e Pseudomonas putida biótipo B também proporcionaram aumento significativo no rendimento de grãos em relação à testemunha. $\mathrm{O}$ tratamento com T. harzianum proporcionou aumento significativo na emergência de plântulas e no rendimento de grãos de milho. Esse aumento foi de $615 \mathrm{~kg} /$ ha acima do rendimento da testemunha, sem tratamento.Em 1998, em Passo Fundo, P. macerans (144), F. oryzihabitanse Agrobacterium radiobacter proporcionaram os melhores aumentos na germinação. Com exceção de $F$. oryzihabitans e de Bacillus subtilis, todos os bioprotetores proporcionaram aumentos significativos no rendimento de grãos. Em Pato Branco, P. putida biótipo B 63, F. oryzihabitans e Pseudominas putida biótipo A (M 970841) proporcionaram as melhores germinações de sementes de milho. Paenibacilus macerans (144), T. harzianum (T-22), F. oryzihabitans, $A$. radiobacter e $B$. subtilis aumentaram significativamente o rendimento de grãos. A microbiolização de sementes apresenta-se como uma alternativa tec nológica para o tratamento de sementes de milho no Brasil.

Palavras-chave: bioproteção, sementes, Zea mays, microbiolização.

\section{ABSTRACT \\ Effect of bioprotectors on seed pathogens, seed emergence, and corn yield}

The use of plant bioprotectors constitutes a modern tactic of seed treatment for corn (Zea mays) disease control without environmental hazards. Experiments were carried out under laboratory and field conditions in Passo Fundo, RS, and in Pato Branco, PR, Brazil, aimed at evaluating the effects of seed microbiolization on seed pathogens, seed emergence, and corn yield. In the laboratory, most bioprotectants significantly reduced pathogen recovery from infected corn seeds. In field experiments, in 1997, in Passo Fundo, only Trichoderma harzianum (T-22) significantly increased seedling emergence and corn yield. All bioprotectors significantly improved seed emergence in Pato Branco. Paenibacillus macerans (144), T. harzianum (T-22), Flavimonas oryzihabitans, and Pseudomonas putida biotype B also provided a significant yield increase over untreated control. T. harzianum treatment showed a yield increase of $615 \mathrm{~kg} / \mathrm{ha}$ over the untreated check. In 1998, in Passo Fundo, P. macerans (144), F. oryzihabitans, and Agrobacterium radiobacter provided the highest increases in seedling germination. All biological treatments, except $F$. oryzihabitans and Bacillus subtilis, showed significant yield increases. In Pato Branco, Pseudomonas putida biotype B, F. oryzihabitans, and P. putida biotype A (M 970841) provided the best corn seed emergence Paenibacillus. macerans (144), T. harzianum (T-22), F. oryzihabitans, A. Radiobacter, and B. subtilis significantly increased grain yield.Seed microbiolization is a promising technological alternative for corn seed treatment in Brazil.

\section{INTRODUÇÃO}

A necessidade da microbiolização de sementes na agricultura mundial tem sido defendida enfaticamente (Luz,

$\overline{* \mathrm{CNPq}}$ fellow
1993, 1996a). Restrições ao uso de fungicidas e os cuidados com o meio ambiente reforçam claramente a necessidade de estudar táticas alternativas, como o uso de bioprotetores. A microbiolização de sementes tem sido realizada com vários patógenos e em várias culturas (Harman, 1991; Kloepper, 1991; Luz, 1991, 1993, 1996a). 
Efeito de bioprotetores em patógenos de sementes e na emergência...

Na cultura de milho, alguns trabalhos foram realizados com resultados positivos (Callan et al., 1990, 1991; Chang \& Kommedahl 1968; Harman et al., 1989; Kommedahl \& Mew, 1975; Luz et al., 1997a, 1997b) no controle de alguns patógenos. No Brasil, os trabalhos iniciais indicaram que alguns bioprotetores apresentam efeitos benéficos significantes na cultura de milho (Luz 1996 a,b, 1998a). O objetivo deste estudo foi examinar os efeitos da microbiolização de sementes no controle de patógenos, na emergência e no rendimento de grãos de milho (Zea mays L.).

\section{MATERIAL E MÉTODOS}

Os experimentos foram realizados em Passo Fundo, RS (Embrapa Trigo), e em Pato Branco, PR (IAPAR). As sementes do híbrido XL 561, suscetível aos patógenos das sementes de milho, usadas neste estudo foram obtidas da empresa Braskalb.

Os tratamentos foram: Paenibacillus macerans (Embrapa Trigo 144), 7,5 g para $100 \mathrm{~kg}$ de sementes, Trichoderma harzianum (Rifai) (Bioworks T-22), $250 \mathrm{~g}$ para $100 \mathrm{~kg}$ de sementes, e Trichoderma virens (Miller, Giddens \& Foster) von Arx (Bioworks G-41), $250 \mathrm{~g}$ para $100 \mathrm{~kg}$ de sementes de trigo, Pseudomonas putida biótipo B (63), Pseudominas putida biótipo A (M 953731 Bg e M 970841), Flavimonas oryzihabitans (M953711), Agrobacterium radiobacter (M953731c), Bacillus subtilis (M972511 e duas bactérias não identificadas (M961111 e M953921, usadas somente em 1997). Para os oito últimos bioprotetores (também da Embrapa Trigo), usaram-se culturas desenvolvidas em 1/4 BDA, por $24 \mathrm{~h}$ a $23 \pm 2{ }^{\circ} \mathrm{C}$. As células foram removidas da superfície do meio de cultura com um pincel e colocadas em água esterilizada, e a concentração de cada antagonista foi de aproximadamente $10^{7}$ unidades formadoras de colônias/ml. Uma suspensão foi, então, aplicada, mergulhando-se as sementes por 3 min e deixandoas secar em temperatura ambiente por $24 \mathrm{~h}$. Uma testemunha sem tratamento, embebida somente em água destilada por 3 min e deixada secar da mesma maneira que as sementes tratadas com os bioprotetores, foi usada para comparação. Para o experimento de laboratório, cada tratamento foi replicado quatro vezes (100 sementes, cinco sementes por placa) e colocado sob luz negra sob fotoperíodo de $12 \mathrm{~h}$ a $24 \pm 2{ }^{\circ} \mathrm{C}$. O arranjo experimental foi em desenho inteiramente casualizado. A presença de fungos patogênicos foi determinada cinco dias após o plaqueamento. Os dados foram expressos em percentagem de cada patógeno nas sementes.

Nos experimentos de campo, as parcelas foram compostas de quatro fileiras de cinco metros cada uma. $\mathrm{O}$ espaçamento entre fileiras foi de um metro, e entre sementes, de 20 centímetros. O delineamento experimental foi de blocos ao acaso, com quatro repetições. A emergência de sementes foi avaliada 30 dias após a semeadura. Os grãos foram colhidos e pesados. Os dados dos tratamentos foram submetidos à análise de variância, e as médias foram separadas, usando-se o teste de LSD de Fisher, ao nível de 0,05.

\section{RESULTADOS E DISCUSSÃO}

Nos experimentos de laboratório, as sementes de milho apresentaram-se severamente contaminadas com os fungos Fusarium graminearum (Schw.), Fusarium moniliforme (Sheldon), Diplodia maydis (Beek) Sacc. e Aspergillus spp., principalmente de A. glaucus (Link ex Gray) e A. flavus (Lk. ex.Fr) (Tabela 1). A incidência de patógenos em 1997 foi superior à de 1998 .

A maioria dos bioprotetores reduziu significativamente o nível de infecção de patógenos nas sementes, destacandose $P$. macerans e $B$. subtilis e os biótipos A e B de $P$. putida, Trichoderma harzianum e T. virens foram particularmente eficientes no controle de $F$. graminearum e de D. maydis, mas não apresentaram eficiência contra $F$. moniliforme e contra Aspergillus spp.

Nos experimentos de campo, no ano de 1997, em Passo Fundo, RS, somente T. harzianum (T-22) aumentou significativamente a emergência e o rendimento de grãos da cultura de milho (Tabela 2). Todos os bioprotetores aumentaram significativamente a emergência de sementes em Pato Branco (PR) (Tabela 3). Paenibacillus. macerans (144), T. harzianum (T-22), F. oryzihabitans e P. putida biótipo B proporcionaram também aumento significativo no rendimento de grãos em relação à testemunha. $\mathrm{O}$ tratamento com $T$. harzianum proporcionou aumento significativo na emergência de plantas e no rendimento de grãos de milho. Quando P. macerans (144) foi usado em Pato Branco, o aumento atingiu $723 \mathrm{~kg} /$ ha acima do rendimento da testemunha, sem tratamento.

Em 1998, em Passo Fundo, P. macerans (144), F. oryzihabitans e $A$. radiobacter proporcionaram os maiores aumentos de emergência (Tabela 2). Com exceção de $F$. oryzihabitans e de $B$. subtilis, os bioprotetores proporcionaram aumentos significativos no rendimento de grãos. Em Pato Branco, $P$. putida biótipo B, $F$. oryzihabitans e $P$. putida biótipo A (M970841) proporcionaram as melhores emergências de sementes de milho (Tabela 3). P. macerans (144), T. harzianum (T-22), F. oryzihabitans, A. radiobacter e $B$. subtilis aumentaram significativamente o rendimento de grãos. Nenhum sintoma de fitotoxidez foi observado no experimento. A ação desses bioprotetores está ligada ao controle de fungos patogênicos de sementes que causam má germinação, início ou aumento de inóculo de patógenos que facilitam o desenvolvimento de doenças em sementes, em plântulas e em planta adulta, reduzindo o rendimento de grãos de milho. Entretanto, o efeito de F. oryzihabitans parece estar ligado a fatores não relacionados com o controle direto de patógenos, uma vez que esse microrganismo não apresentou eficiência contra os fungos patogênicos (Tabela 1). Mecanismos de ação, tais como indução de resistência, fitohormônios, mineralização de nutrientes e outros, podem estar envolvidos (Boronin et al., 1993; Liu et al., 1995 a,b; Kloepper et al., 1992; Luz, 1996a). Esses mecanismos de ação também podem estar agindo em interação com o 
W.C. Luz

TABELA 1 - Efeito de bioprotetores aplicados em sementes de milho (Zea mays) sobre a redução de patógenos. Passo Fundo, RS

\begin{tabular}{|c|c|c|c|c|c|c|c|c|}
\hline \multirow{3}{*}{ Tratamento } & \multicolumn{8}{|c|}{ Percentagem de patógenos nas sementes * } \\
\hline & \multicolumn{2}{|c|}{$\begin{array}{c}\text { Fusarium } \\
\text { graminearum }\end{array} * *$} & \multicolumn{2}{|c|}{ Diplodia maydis } & \multicolumn{2}{|c|}{$\begin{array}{c}\text { Fusarium } \\
\text { moniliforme }\end{array}$} & \multicolumn{2}{|c|}{ Aspergillus spp. } \\
\hline & 1997 & 1998 & 1997 & 1998 & 1997 & 1998 & 1997 & 1998 \\
\hline Testemunha & $22 \mathrm{c}$ & $11 \mathrm{~d}$ & $14 \mathrm{c}$ & $10 \mathrm{~d}$ & $31 \mathrm{~b}$ & $2 \mathrm{~b}$ & $22 \mathrm{c}$ & $4 \mathrm{c}$ \\
\hline Agrobacterium radiobacter & $20 \mathrm{c}$ & $11 \mathrm{~d}$ & $4 \mathrm{~b}$ & $0 \mathrm{a}$ & $30 \mathrm{~b}$ & $2 \mathrm{~b}$ & $22 \mathrm{c}$ & $4 \mathrm{c}$ \\
\hline Bacillus subtilis (em 1998) & - & $0 \mathrm{a}$ & - & $0 \mathrm{a}$ & - & $0 \mathrm{a}$ & - & $1 \mathrm{a}$ \\
\hline Flavimonas oryzihabitans & $20 \mathrm{c}$ & $11 \mathrm{~d}$ & $0 \mathrm{a}$ & $8 \mathrm{~d}$ & $29 \mathrm{~b}$ & $2 \mathrm{~b}$ & $19 \mathrm{c}$ & $3 \mathrm{c}$ \\
\hline M 9611.1.1. B (em 1997) & $22 \mathrm{c}$ & - & $2 b$ & - & $30 \mathrm{~b}$ & - & $22 \mathrm{c}$ & - \\
\hline M9539.2.1 lev (em 1997) & $21 \mathrm{c}$ & - & $2 \mathrm{~b}$ & - & $30 \mathrm{~b}$ & - & $22 \mathrm{c}$ & - \\
\hline Paenibacillus macerans (144) & $4 \mathrm{a}$ & $5 \mathrm{~b}$ & $0 \mathrm{a}$ & $2 \mathrm{~b}$ & $15 \mathrm{a}$ & $0 \mathrm{a}$ & $4 \mathrm{a}$ & $0 \mathrm{a}$ \\
\hline Pseudomonas putida biót A (M953731Bg) & $6 \mathrm{~b}$ & $7 \mathrm{c}$ & $0 \mathrm{a}$ & $8 \mathrm{~d}$ & $15 \mathrm{a}$ & $0 \mathrm{a}$ & $11 \mathrm{~b}$ & $4 \mathrm{c}$ \\
\hline P. putida biót A(M970841, em 1998) & - & $7 \mathrm{c}$ & - & $4 \mathrm{c}$ & - & $2 \mathrm{~b}$ & - & $2 \mathrm{~b}$ \\
\hline P. putida biót $\mathrm{B}(63)$ & $6 \mathrm{~b}$ & $7 \mathrm{c}$ & $2 \mathrm{~b}$ & $2 \mathrm{~b}$ & $16 \mathrm{a}$ & $0 \mathrm{a}$ & $12 \mathrm{~b}$ & $3 \mathrm{c}$ \\
\hline Trichoderma harzianum (T-22) & $2 \mathrm{a}$ & $0 \mathrm{a}$ & $0 \mathrm{a}$ & $0 \mathrm{a}$ & $31 \mathrm{~b}$ & $2 \mathrm{~b}$ & $22 \mathrm{c}$ & $4 \mathrm{c}$ \\
\hline T. virens $(\mathrm{G}-41)$ & $2 \mathrm{a}$ & $0 \mathrm{a}$ & $0 \mathrm{a}$ & $2 \mathrm{~b}$ & $31 \mathrm{~b}$ & $2 \mathrm{~b}$ & $21 \mathrm{c}$ & $4 \mathrm{c}$ \\
\hline $\mathrm{CV} \%$ & 4.7 & 5.2 & 6.1 & 2.3 & 9.4 & 2.1 & 9.2 & 2.1 \\
\hline
\end{tabular}

*Média de 4 repetições. Dados seguidos por letras iguais nas colunas não diferem entre si, de acordo com o teste de LSD de Fisher, a p=0,05.

TABELA 2 - Efeito do tratamento de sementes com bioprotetores na emergência (\%) e no rendimento de grãos (kg/ha) de milho (Zea mays). Embrapa Trigo, Passo Fundo, RS

\begin{tabular}{|c|c|c|c|c|}
\hline \multirow{2}{*}{ Tratamento } & \multicolumn{2}{|c|}{ Emergência (\%)* } & \multicolumn{2}{|c|}{ Rendimento (kg/ha)* } \\
\hline & 1997 & 1998 & 1997 & 1998 \\
\hline Testemunha & $78 \mathrm{~b}$ & $89 \mathrm{c}$ & $7.268 \mathrm{~b}$ & $4.966 \mathrm{c}$ \\
\hline Agrobacterium radiobacter (M953731C) & $79 \mathrm{~b}$ & $94 \mathrm{a}$ & $7.300 \mathrm{~b}$ & $5.286 \mathrm{ab}$ \\
\hline Bacillus subtilis (M952511, em 1998) & - & $91 \mathrm{bc}$ & - & $5.141 \mathrm{bc}$ \\
\hline Flavimonas oryzihabitans(M53711) & $80 \mathrm{~b}$ & $93 \mathrm{a}$ & $7.295 \mathrm{~b}$ & $5.141 \mathrm{bc}$ \\
\hline M 961111 (em 1997) & $78 \mathrm{~b}$ & - & $7.275 \mathrm{~b}$ & - \\
\hline M953921 lev (em 1997) & $78 \mathrm{~b}$ & - & $7.273 \mathrm{~b}$ & - \\
\hline Paenibacillus macerans (144) & $80 \mathrm{~b}$ & $94 \mathrm{a}$ & $7.292 \mathrm{~b}$ & $5.335 \mathrm{a}$ \\
\hline Pseudomonas putida biót A (M953731Bg) & $80 \mathrm{~b}$ & $90 \mathrm{c}$ & $7.340 \mathrm{~b}$ & $5.283 \mathrm{ab}$ \\
\hline Ps putida biót A (M970841) (em 1998) & - & $92 \mathrm{~b}$ & - & $5.213 \mathrm{ab}$ \\
\hline P. putida biót $\mathrm{B}(63)$ & $79 \mathrm{~b}$ & $92 \mathrm{~b}$ & $7.313 \mathrm{~b}$ & $5.302 \mathrm{a}$ \\
\hline Trichoderma harzianum (T-22) & $88 \mathrm{a}$ & $91 \mathrm{bc}$ & $7.883 \mathrm{a}$ & $5.318 \mathrm{a}$ \\
\hline T. virens $(\mathrm{G}-41)$ & $83 \mathrm{~b}$ & $91 \mathrm{bc}$ & $7.301 \mathrm{~b}$ & $5.350 \mathrm{a}$ \\
\hline C.V.\% & 10,3 & 11,3 & 9,3 & 13,5 \\
\hline
\end{tabular}

*Médias seguidas da mesma letra, na coluna, não diferem entre si, pelo teste de LSD de Fisher, a 5\% de probabilidade.

controle direto de patógenos para os outros bioprotetores (Luz, 1996a).

Nos experimentos, alguns bioprotetores presentes controlaram os patógenos de sementes, melhoraram a emergência de plântulas e aumentaram o rendimento de grãos de milho. Outros estudos apresentaram evidências similares usando $P$. macerans, $P$. putida biótipo B e outros bioprotetores (Luz, 1996 a, b; Luz 1998a; Luz et al., 1997 a,b). Benefícios semelhantes foram mostrados com T. harzianum (Harman et al., 1989; Luz, 1998a) e com B. subtilis (Chang \& Kommedahl, 1968).

O tratamento de sementes de milho com fungicidas no Brasil é uma prática usada para protegê-las contra as podridões de sementes, morte e tombamento de plantas induzidos por fungos na semente ou no solo (Luz, 1997a). A maioria dos híbridos comercializados são previamente tratados com fungicidas.

Vários fungicidas têm sido investigados com resultados de eficiência contra os principais patógenos de milho (Luz 1996c,d, 1997a,b,c, 1998b; Luz \& Pereira, 1998). Contudo, há necessidade de usar produtos biológicos que sirvam como alternativa e apresentem controle dos principais patógenos e aumento de rendimento de grãos da cultura. Por esse motivo, os resultados mostrados no presente estudo demonstraram que os bioprotetores apresentam-se como uma tecnologia alternativa para o tratamento de sementes de milho. Esses bioagentes poderão ter um importante impacto na redução do uso excessivo de fungicidas, no alcance da agricultura sustentável e na proteção do ambiente. 
Efeito de bioprotetores em patógenos de sementes e na emergência...

TABELA 3 - Efeito do tratamento de sementes com bioprotetores na emergência (\%) e no rendimento de grãos (kg/ ha) de milho (Zea mays), Embrapa Trigo, Pato Branco, PR

\begin{tabular}{|c|c|c|c|c|}
\hline \multirow{2}{*}{ Tratamento } & \multicolumn{2}{|c|}{ Emergência (\%)* } & \multicolumn{2}{|c|}{ Rendimento (kg/ha)* } \\
\hline & 1997 & 1998 & 1997 & 1998 \\
\hline Testemunha & $70 \mathrm{~d}$ & $83 \mathrm{~cd}$ & $5.462 \mathrm{c}$ & $5.832 \mathrm{c}$ \\
\hline Agrobacterium radiobacter (M953731C) & $82 \mathrm{~b}$ & $85 \mathrm{bc}$ & $5.669 \mathrm{bc}$ & $6.632 \mathrm{a}$ \\
\hline Bacillus subtilis (M952511,em 1998) & - & $83 \mathrm{~cd}$ & - & $6.772 \mathrm{a}$ \\
\hline Flavimonas oryzihabitans (M53711) & $80 \mathrm{bc}$ & $86 \mathrm{ab}$ & $6.035 \mathrm{a}$ & $6.700 \mathrm{a}$ \\
\hline M 961111 (em 1996/97) & $82 \mathrm{~b}$ & - & $5.684 \mathrm{bc}$ & \\
\hline M953921 lev (em 1997) & $78 \mathrm{c}$ & - & $5.678 \mathrm{bc}$ & - \\
\hline Paenibacillus macerans (144)86 A & $86 \mathrm{a}$ & $83 \mathrm{~cd}$ & $6.185 \mathrm{a}$ & $6.542 \mathrm{ab}$ \\
\hline Pseudomonas putida biót A (M953731Bg) & $77 \mathrm{c}$ & $85 \mathrm{bc}$ & $5.771 \mathrm{bc}$ & $5.911 \mathrm{c}$ \\
\hline P. putida biót A (M970841) (em 1998) & - & $86 \mathrm{ab}$ & - & $5.892 \mathrm{c}$ \\
\hline P. putida biót $\mathrm{B}(63)$ & $81 \mathrm{bc}$ & 88 a & $5.875 \mathrm{~b}$ & $6.100 \mathrm{c}$ \\
\hline Trichoderma harzianum (T-22) & $79 \mathrm{c}$ & $83 \mathrm{~cd}$ & $6.040 \mathrm{a}$ & $6.500 \mathrm{ab}$ \\
\hline T. virens $(\mathrm{G}-41)$ & $79 \mathrm{c}$ & $84 \mathrm{~cd}$ & $5.623 \mathrm{bc}$ & $5.832 \mathrm{c}$ \\
\hline C.V.\% & 12,4 & 10,8 & 12,3 & 14,4 \\
\hline
\end{tabular}

*Médias seguidas da mesma letra, na coluna, não diferem entre si, pelo teste de LSD de Fisher, a $5 \%$ de probabilidade.

\section{REFERÊNCIAS BIBLIOGRÁFICAS}

BORONIN, A.M., KOCHETKOV, V.V., DUBEIKOVSKI, A.N. \& MORDUKHOVA, E.A. Biological control of soilborne plant pathogens by PGPR Pseudomonas isolated in Russia. Resumos, $7^{\circ}$ International Congress of Plant Pathology, Montreal, Canada, International Society of Plant Pathology. 1993. p. 276.

CALLAN, N.W., MATHRE, D.E. \& MILLER, J.B. Biopriming seed treatment for biological control of Pythium ultimum preemergence damping-off in sh2 sweet corn. Plant Disease. 74:368-372. 1990.

CALLAN, N.W., MATHRE, D.E. \& MILLER, J.B. Field performance of sweet corn seed bio-primed and coated with Pseudomonas fluorescens AB254. HortScience 26:1163-1169. 1991.

CHANG, I. \& KOMMEDAHL, T. Biological control of seedling blight of corn by coating kernels with antagonistic microorganisms. Phytopathology 58:13951401. 1968.

HARMAN, G.E. Deployment tactics for biocontrol agents in plant pathology. In: BAKER, R. \& DUNN, P.E. (Eds.) New directions in biological control. Alternatives for suppressing agricultural pests and diseases. New York, Liss., 1989. pp.779-792.

HARMAN, G.E. Seed treatments for biological control of plant disease. Crop Protection 10:166-71. 1991.

KLOEPPER, J.W. Plant growth-promoting rhizobacteria as biological control agents of soilborne diseases. In: The biological control of plant diseases. Ed. J. BAYPETERSON (Ed.). Taiwan, Food and Fertilizer Technological Center. 1991. pp. 142-152.

KOMMEDAHL, T. \& MEW, I. C. Biocontrol of corn root infection in the field by seed treatment with antagonists. Phytopathology 65: 296-300. 1975.

LIU, L., KLOEPPER, J.W. \& TUZUN, S. Induction of systemic resistance in cucumber against bacterial angular leaf spot by plant growth-promoting rhizobacteria. Phytophatology 85:843-847. 1995a.

LIU, L., KLOEPPER, J.W. \& TUZUN, S. Induction of systemic resistance in cucumber against Fusarium wilt by plant growth-promoting rhizobacteria. Phytophatology 85:695-698. 1995b.

LUZ, W.C. da. Controle biológico das doenças na espermosfera. In: Controle biológico de doenças de plantas. Jaguariúna, EMBRAPA-CNPDA, 1991. pp. 2531.

LUZ, W.C. da. Microbiolização de sementes para o controle das doenças das plantas. In: LUZ, W.C. da, FERNANDES, J.M.C., PRESTES, A.M. \& PICININI, E.C. (Eds.). Revisão Anual de Patologia de Plantas, v.1, 1993. pp. 33-77.

LUZ, W. C. da. Rizobactérias promotoras de crescimento de plantas e bioproteção. In: LUZ, W.C. da, FERNANDES, J.M.C. \& PRESTES, A.M. \& PICININI, E.C. (Eds.). Revista Anual de Patologia de Plantas, v. 4. Passo Fundo, RS. 1996a. pp.1-47.

LUZ, W.C. da. Efeito de rizobactérias promotoras de crescimento de plantas de trigo e de milho. Fitopatologia Brasileira 21:434. 1996b.

LUZ, W.C. da. Tratamento de sementes de milho com fungicidas. Circular Técnica no 7. Embrapa Trigo. 1996c.

LUZ, W.C. da. Espectro de ação de novos fungicidas para tratamento de sementes de milho e seus efeitos no rendimento. Fitopatologia Brasileira, 21:369. 1996d.

LUZ, W.C. da. Espectro de ação de fungicidas contra fungos de sementes de milho. Resumo, $21^{\circ}$ Congresso Nacional de Milho e Sorgo. Londrina, PR. Resumos. Londrina: IAPAR, p.288. 1996a.

LUZ, W.C. da. Tratamento de sementes de milho com fungicidas. Circular Técnica no 7. $2^{\mathrm{a}}$ edição. Embrapa 
Trigo. 24p. 1997a.

LUZ, W.C. da. Evaluation of seed treatment fungicides for emergence and yield of corn. Fungicide \& Nematicide Tests 52:303. 1997b.

LUZ, W.C. da. Effect of seed treatment on corn pathogen control, stand, and yield. Fungicide \& Nematicide Tests, 52:303. 1997c.

LUZ, W.C. da. Efeito de bioprotetores na germinação e no rendimento de grãos de milho. Anais, $21^{\circ}$ Congresso Nacional de Milho e Sorgo, Recife, PE. 1998a. p.227.

LUZ, W.C. da. Efficacy of chemical seed treatments in controlling seed decay of corn. Fungicide \& Nematicide
Tests 53:390. 1998b.

LUZ, W.C. da \& PEREIRA, L.R. Tratamento de sementes com fungicidas relacionado com o controle de patógenos e rendimento de milho. Ciência Rural 28:537-541. 1998.

LUZ, W.C. da., BERGSTRON, G.C. \& STOCKWELL, C.A. Seed microbiolization for control of Fusarium species in cereais. Phytopathology 87:522. 1997a. (Abstract).

LUZ, W.C. da, BERGSTRON, G.C. \& STOCKWELL, C.A. Seed bioprotectants for control of Fusarium on wheat and corn. Proceedings of the First National Head Blight Forum. St. Paul, Mn, USA. 1997b. 